\title{
Entre comportamientos éticos heterónomos y autorregulación en escenarios de universidad: un debate permanente
}

Between heteronomous ethical behaviors and self-regulation in university settings:

a permanent debate

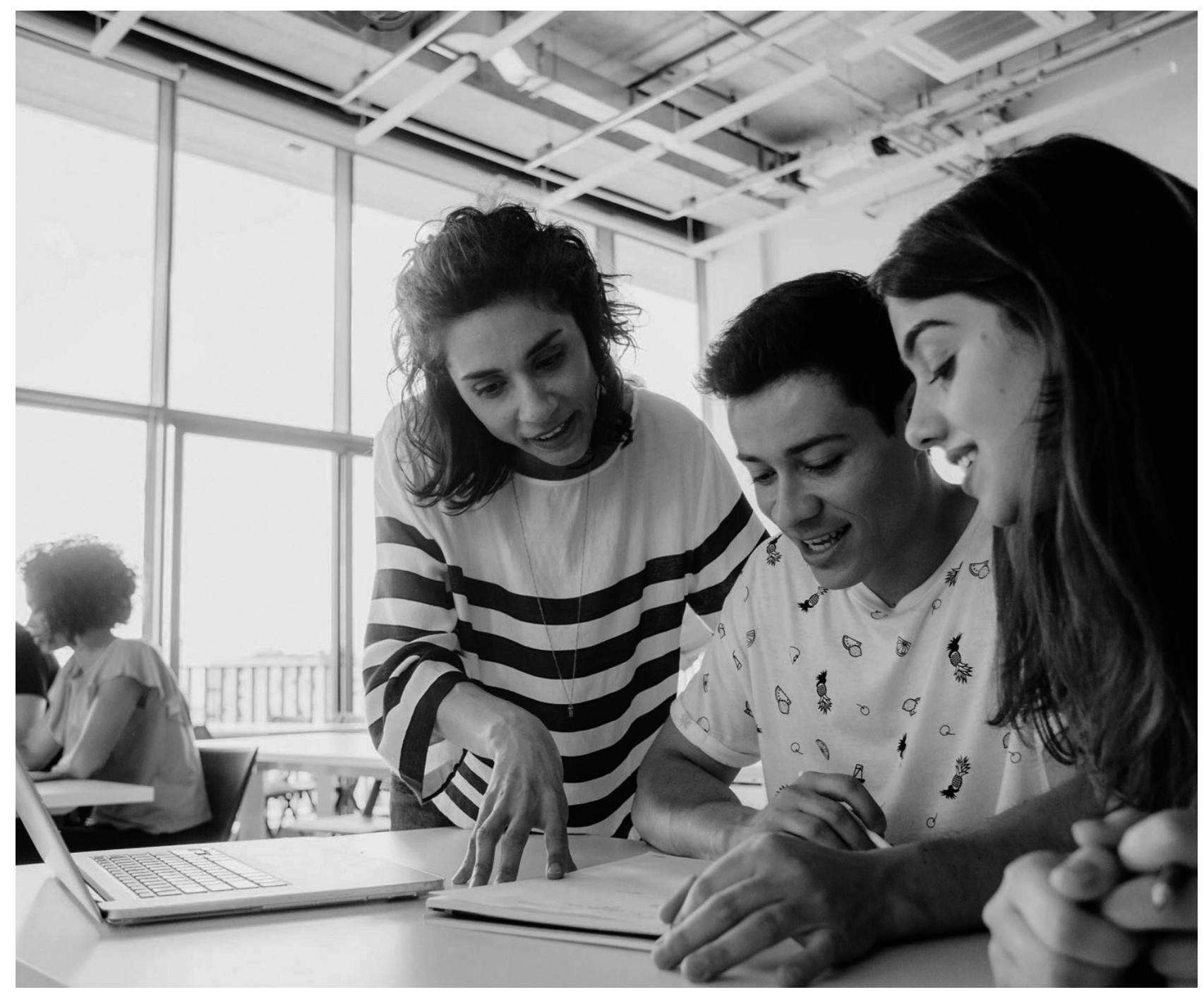




\title{
Entre comportamientos éticos heterónomos y autorregulación en escenarios de universidad: un debate permanente ${ }^{1}$
}

\section{Between heteronomous ethical behaviors and self-regulation in university settings: a permanent debate}

\author{
Juan José Burgos Acosta²
}

Artículo recibido en julio de 2017; artículo aceptado en septiembre de 2017.

\begin{abstract}
Este artículo puede compartirse bajo la Licencia Creative Commons Atribución-No Comercial-Compartir lgual 2.0 Genérica y se referencia usando el siguiente formato: Burgos, J. (2018). Entre comportamientos éticos heterónomos y autorregulación en escenarios de universidad: un debate permanente. I+D Revista de Investigaciones, 11(1), 56-65.
\end{abstract}

DOI: https://doi.org/10.33304/revinv.v11n1-2018005

\begin{abstract}
Resumen
Este trabajo de investigación estuvo dirigido a estudiantes de tres facultades de pregrado de una universidad con el propósito de determinar las relaciones entre ética y autorregulación en los procesos de aprendizaje. Se empleó una metodología cuantitativa, mediante la aplicación del cuestionario y el uso del paquete estadístico SPSS que arrojó resultados como que el grueso de la población encuentra felicidad en lo que está estudiando, lo cual deviene en que cuando se disfruta lo que se hace los niveles de coherencia entre el decir y el hacer se estrechan; sufren más cuando tienen que responder cuestionarios memorísticos que los encasillan y los suelen llevar a comportamientos antiéticos, como hacer trampa en un examen o plagiar un trabajo; la valoración hecha sobre la relación entre formación en la universidad y ética tiende hacia lo positivo, y la autorregulación fue considerada como un elemento importante en el aprendizaje y los valores éticos.
\end{abstract}

Palabras claves: autorregulación, ética, aprendizaje, comportamiento.

\begin{abstract}
This research was aimed at students of three undergraduate faculties of a university with the purpose of determining the relationships between ethics and self-regulation in the learning processes. A quantitative methodology was used, through the application of the questionnaire and the use of the statistical package SPSS that yielded results such as: The bulk of the population finds happiness on what they are studying, it reflects on the fact that when you enjoy what you do levels of coherence between saying and doing becomes narrower. They suffer more when they have to answer questionnaires that constrain and often lead to unethical behaviors, such as cheating on an exam or plagiarize a job. Assessment on the relationship between university training and ethics that tends toward the positive was carried out. Self-regulation was considered as an important element for the learning process and for ethical values.
\end{abstract}

Keywords: self-regulation, ethics, learning, behavior.

1. Artículo resultado de un proyecto de investigación culminado, de enfoque cuantitativo.

2. Licenciado en Filosofía, Universidad Santo Tomás. Licenciado en Teología, Universidad Javeriana. Posdoctorado en Educación, Ciencias Sociales e Interculturalidad, Universidad Santo Tomás. Doctor en Educación Universidad Santo Tomás. Doctor HC en Filosofía de la Educación otorgado por el Consejo Iberoamericano. Magister en Educación, Universidad Javeriana. Magister en Estudios Políticos, Universidad Javeriana. Profesor de planta, Universidad Colegio Mayor de Cundinamarca de la ciudad de Bogotá (Colombia). ORCID ID: https://orcid.org/ 0000-0002-6066. Correo electrónico institucional: juanj.burgos@unicolmayor.edu.co. 


\section{Introducción}

A medida que pasa el tiempo, el acumulado del conocimiento es cada vez más asombroso en todos los campos del saber gracias al empuje que continuamente recibe de la investigación. Este trabajo, que constituye un aporte más a esos avances del conocimiento en el campo de la pedagogía, permitió establecer -desde la mirada del investigador con base en los resultados "objetivos" que se obtuvieron de los participantes- una comprensión de las relaciones entre autorregulación, aprendizaje y ética, con el propósito de que se siga investigando alrededor del problema propuesto.

\section{Tendencias de los estudios y reflexiones teóricas respecto del fenómeno}

De acuerdo con los avances recabados en torno al problema de investigación, se puede considerar que existen más coincidencias que distanciamientos en los trabajos que se pudieron consultar. Existe una relación estrecha entre aprendizaje, autorregulación y comportamiento ético, al menos en algunos trabajos. El aprendizaje es una forma de acontecimiento ético en la medida en que abre un camino de transformación que permite al sujeto tomar conciencia para crear puentes de apertura hacia el otro dentro de un contexto, aspecto que el profesor Habermas (2008), cuando desarrolla el debate en su tesis sobre conciencia moral y acción comunicativa, denominó procesos intersubjetivos. En este sentido, se destacan también estudios que devienen en la formación de valores específicos que deben enseñarse en la educación superior, teniendo en cuenta que las sociedades modernas, además de ser complejas, son multiculturales y en ellas los procesos de enseñanza y aprendizaje revisten aspectos diferenciados que se reflejan en el análisis de resultados cuando los grupos son intervenidos durante determinados tiempos. Hay que señalar, sin embargo, que los estudios en este sentido son escasos (García \& González, 2015).

Ahora bien, este trabajo se compone de cinco apartados: en el primero se presentan los antecedentes que recogen los avances en el tema problema planteado y las tendencias de los estudios encontrados. En el segundo se ofrece la descripción del planteamiento del problema, el cual termina en la pregunta que se consideró para guiar toda la indagación. En el apartado tercero se propone la metodología con el tipo de investigación, el universo de la población y las técnicas de recolección de la información. En la parte cuarta se describen los resultados o hallazgos del trabajo de investigación. Finalmente, se proponen algunas conclusiones orientadoras. La mayoría de los estudios insisten, dentro de las conclusiones y proyecciones, en la necesidad de superar las formas conservadoras de la enseñanza heredadas de una tradición que muchas veces se ha anquilosado, manifestando el temor a lo desconocido y a la misma incertidumbre, impidiendo de esa manera generar espacios democráticos de formación que ayudarían significativamente a fortalecer un aprendizaje autorregulado bajo principios éticos, tal como lo ha advertido la profesora e investigadora neoyorquina Nussbaum (2010) al referirse a la necesidad de una educación fuertemente moral, que siembre la autonomía y la capacidad crítica de los estudiantes, dado que son los que, finalmente, serán lo que ella denomina "ciudadanos del mundo".

En esa línea de relación se encuentran también varios estudios que destacan como un hecho real la poderosa influencia de la sociedad del conocimiento como cuarto orden de la economía, según lo proponen autores como Maldonado (2005), quien ha llevado a que se realicen investigaciones en las cuales se han propuesto una serie de estrategias tendientes a evaluar, desde diferentes metodologías, las relaciones entre motivación, autorregulación y aprendizaje a través del monitoreo de programas semestrales y anuales en grupos de estudiantes universitarios con muestras significativas, cuyos resultados han ayudado al avance en la comprensión del fenómeno.

Como fuente principal en las investigaciones que se han realizado referentes a la autorregulación en educación, todos los estudios hechos en terreno recurren a Zimmerman (1989), quien, entre otras cosas, destaca la inevitable relación entre motivación, conducta y contexto como una tríada en la cual el estudiante actúa como sujeto activo y el maestro, como mediador y facilitador que constantemente está llamado a innovar para fortalecer la autonomía de los individuos, y, en esa medida, obtener resultados académicos eficientes.

Otros estudios consultados muestran que existe una relación muy importante entre planificación, seguimiento y evaluación en las dinámicas de aprendizaje que determinan lo que han denominado "el aprender a aprender". Sin embargo, muchas veces las incertidumbres y los resultados adversos frente a metas propuestas o esperadas pueden aprovecharse para innovar y proponer nuevas alternativas de aprendizaje que respondan a los vacíos encontrados, lo cual estaría de acuerdo con las orientaciones de Morín (1999), al sugerir la necesidad de apropiarse de "la incertidumbre en el conocimiento" como uno de los sietes saberes claves para la educación en la era posmoderna, sin el cual no es posible enfrentar las complejidades culturales y las responsabilidades como ciudadanos (Serrano, 2015). 
Finalmente, se destaca la fuerte tendencia a incorporar en los estudios consultados dos grandes categorías trasversales que delimitan algunos trabajos durante el ejercicio de indagación: el aprendizaje profundo y el aprendizaje superficial, "probando" muchas veces la hipótesis de que ambas dimensiones son directamente proporcionales a los niveles altos, medios o bajos de autorregulación de la población estudiantil, no sin dejar pasar el hecho de que existen factores multisistémicos como el contexto, las creencias y el rol del profesor, que juegan papeles fundamentales en el proceso, coincidiendo de esa manera con los planteamientos de autores como Vasco (2003), para quien los resultados que se puedan obtener en la educación dependen de las relaciones transdisciplinares y del tejido cultural que se establezca.

De acuerdo con las características de los antecedentes antes descritos, esta investigación pretende continuar aportando a la comprensión del fenómeno, especialmente desde las relaciones entre la dimensión ética y la autorregulación, pues, como se pudo notar, los avances que abordan esa articulación son escasos; se ha trabajado más la autorregulación en relación con el aprendizaje y el rendimiento académico, con todas sus variantes.

\section{Planteamiento del problema}

La universidad está llamada no solo a reconocer que evidentemente existen problemas sociales, políticos, económicos y ecológicos que afectan la calidad de vida y la dignidad de las personas, sino también a realizar constantemente ejercicios de investigación que devengan en acciones que contribuyan a transformar los paradigmas educativos sobre el aprendizaje que se produce en los distintos campos que surgen de la economía del conocimiento. No obstante, las consideraciones anteriores adquieren más relevancia si la universidad se piensa también desde dentro, en términos de sus propias dinámicas y las apuestas metodológicas que la identifican.

Es claro que los estudiantes de las distintas facultades, incluso antes de haber ingresado a cualquier programa, ya vienen con unos comportamientos y creencias culturales que determinan en gran medida el desarrollo de su proceso de aprendizaje. En ese sentido, comparten el conflicto básico que viven los seres humanos como resultado de la oposición de dos redes de conversaciones o metaculturas (Maturana \& VerdenZöller 2003; Echeverría, 2010; Pérez, 2010: Vives, (2015), las cuales han configurado al mismo tiempo la cultura ${ }^{3}$ solidaria, caracterizada por el consenso, la cooperación y la asunción de ciertos valores como modos de vida; y la cultura patriarcal, caracterizada por la imposición, el autoritarismo, la apropiación, la competencia y el consumismo. Este modo de relacionarse, que es el que está más acentuado históricamente, se sitúa dentro del paradigma según el cual la vida se construye como una continua competencia por la dominación y el control sobre el mundo y sobre los otros.

El continuo choque de esas dos culturas, la patriarcal y la solidaria, es lo que hace que el ser humano en la vida cotidiana constantemente se vea abocado a problemas, no solamente de convivencia, sino también de las formas en que asume los procesos de aprendizaje dentro de las estructuras académicas institucionalizadas, como es la universidad. Esto, en tanto, por un lado, desde el campo de la convivencia, el individuo desea participar, cooperar, ser solidario, vivir democráticamente, etc., pero, al mismo tiempo se comporta de maneras excluyentes y agresivas. $Y$ por otro, esas mismas tensiones, contradicciones y conflictos que produce la confrontación de dichas culturas es lo que los estudiantes universitarios también manifiestan en los escenarios de formación en aspectos como la responsabilidad en las tareas, el rendimiento académico, las formas de asumir la evaluación, los hábitos de estudios y las relaciones con los maestros. De acuerdo con ello, el escenario formativo entra constantemente en conflicto cuando se encuentra que los resultados académicos no son los que inicialmente se esperaban, a pesar de que han sido bien planeados en cada uno de los programas que conforman el proyecto educativo de la institución.

Al respecto, los estudios realizados en algunos países de Europa y América Latina, y en Colombia (Tejedor, 2006; Garbanzo, 2007; Di Gresia, 2007; Martín, 2007; Camarero, 2014; Albornoz, 2009; Erazo, 2012; Salcedo, 2010; Garzón, Rojas, et al., 2010), entre otros, a pesar de los contextos diferenciados, coinciden en algunas características comunes que afectan el rendimiento académico y que están relacionados directamente con la autorregulación de los estudiantes universitarios. Aunado a ello, existen factores institucionales, profesorales y de las dinámicas propias de cada estudiante que hay que tener en cuenta a la hora de valorar y comprender la manera como se asume el aprendizaje; entre ellos, los estudios mencionados destacan: los estudiantes llegan a la universidad sin hábitos y disciplina de estudio. Muchos de ellos se ven obligados a estudiar más por necesidad que por gusto. De otra parte, el facilismo de la vida actual los lleva a un aprendizaje superficial, en gran parte porque

\footnotetext{
3. El concepto de cultura aquí se aborda desde los planteamientos de Maturana \& Verden-Zöller (2003) y Geertz (2005), quienes la definen como una red de conversaciones con múltiples significados que define y constituye todo el quehacer de una comunidad humana, el cual se expresa en un continuo fluir en el lenguaje y las emociones que permiten coordinar ciertas acciones dentro de un contexto.
} 
el uso inadecuado de las tecnologías y la información que circula por redes sociales buscan más construir relaciones culturales de amistad, música o actualidad consumista, que apropiarse de la circulación académica de calidad que permita el debate y la profundización de estudios e investigación actualizada y fundamentada.

También, muchos estudiantes perciben la normatividad académica más como un requisito que escasamente se cumpleque como un medio para formarse profesionalmente. Otra coincidencia para destacar es que las pruebas internacionales y nacionales muestran notables vacíos que se repiten constantemente, $y$ ello se debe, en gran parte, a que no existen políticas públicas que incentiven una educación que requiere inversión económica y acompañamiento constante, tanto en calidad como en cobertura. Otros factores son: el sufrimiento que puede producir preparar una evaluación y obtener resultados favorables, los métodos de enseñanza usados por algunos profesores, que no garantizan éxito académico. Asimismo, trabajar en el día y estudiar en la noche marca diferencias sustanciales en las formas de aprender y de evaluar los procesos.

Sin embargo, más allá de los resultados académicos, existe un vacío importante que es necesario indagar con respecto a las relaciones entre el comportamiento ético y su incidencia en el aprendizaje autorregulado, dado que a partir del análisis de los resultados se pueden establecer lineamientos que eventualmente sirven como herramientas metodológicas para implementar estrategias que contribuyan a mejorar los procesos de aprendizaje autorregulado.

Las consideraciones anteriores dieron paso a la pregunta que orientó toda la investigación: ¿cuáles son las relaciones entre comportamientos éticos heterónomos y autorregulación del aprendizaje en estudiantes de las facultades de Educación, Economía y Contaduría de la Universidad la Gran Colombia?

Para resolver esta interrogante se propuso como objetivo general determinar las relaciones entre comportamiento ético y aprendizaje autorregulado de los estudiantes de la Facultad de Educación, Economía y Contaduría de la Universidad la Gran Colombia, y como específicos, describir características de comportamiento ético en los estudiantes, conocer cómo se autorregulan los estudiantes en el proceso de aprendizaje y establecer las relaciones entre motivación y autorregulación que manifiestan los estudiantes.

\section{Metodología}

El enfoque metodológico se asumió en el marco de una postura epistemológica no positivista que Páramo (2010) denomina alternativa, mostrando que existen una variedad de teorías sobre cómo se construye el conocimiento que ha venido surgiendo a lo largo del tiempo y que indudablemente han contribuido al avance de la investigación. En este sentido, existen tres aspectos fundamentales que en esta investigación se tuvieron en cuenta respecto de cómo se construye el conocimiento en el proceso de investigación (Ibañez, 2014). El primero tiene que ver con el papel del sujeto u observador frente al fenómeno u objeto que desea investigar. El segundo, la manera como se conoce e interpreta la "realidad" que se va a investigar; y el tercero, cuál es el camino que permite a través de una estructura coherente y pertinente alcanzar cierto grado de "objetividad" que sea tan confiable que permita avanzar en la comprensión del objeto estudiado, en este caso las relaciones entre comportamiento ético y autorregulación en estudiante de la Universidad la Gran Colombia.

\section{Tipo de investigación}

De acuerdo con el enfoque metodológico que se ha descrito anteriormente, el tipo de investigación que se asumió es de carácter cuanti/cuali, en tanto la mayoría de las escuelas de investigación (Sampieri, Collado \& Lucio, 2006; Pereira, 2010; Páramo, 2010; Cascante, 2011, entre otros) consideran que permite combinar elementos cualitativos y cuantitativos y generar procesos de triangulación complementaria que favorecen una mejor comprensión de los fenómenos socioeducativos que se estudian.

\section{Universo de la población}

La investigación tuvo en cuenta una muestra de 160 estudiantes de la jornada nocturna, hombres y mujeres de tres facultades de pregrado: Contaduría, Economía y Educación. La muestra, que se tomó en distintos semestres en cada facultad, comprende edades entre 18 y 30 años.

\section{Técnicas de recolección de la información}

La técnica de recolección de información empleada fue el cuestionario, el cual, según autores como Páramo "es una de las técnicas de recolección de datos más utilizadas en la investigación social debido ignorar fundamentalmente, a que a través de ésta se puede recoger gran cantidad de datos, sobre actitudes, intereses y comportamientos" (2010). Para mantener la coherencia, tanto con el enfoque metodológico como con el tipo de investigación, se tuvo en cuenta el aporte de un cuestionario que ya se ha puesto en práctica: el CEAM II, traducción y adaptación del Motivational Strategies Learning Questionnaire [MSLQ] de Pintrich, Smith, García y Mckeachie (1991). El MSLQ se 
fundamenta en el modelo de aprendizaje autorregulado de Mckeachie, Pintrich y colaboradores (Mckeachie, Pintrich, Lin y Smith, 1986). Este modelo integra diversos factores que influyen en el aprendizaje y pone el énfasis en los factores cognitivos / motivacionales y en sus relaciones, así como en la influencia que tienen en la implicación del estudiante en su aprendizaje y en su rendimiento académico.

\section{Resultados}

Aunque existen patrones comunes que influyen en las dinámicas de autorregulación, como, por ejemplo, el tipo de evaluación, la didáctica, el rol del profesor, etc., en todo caso esta es en cada individuo, en razón a factores biológicos, culturales y familiares que cada uno porta en su proceso de formación integral (Campo y Restrepo, 2008). En ese sentido, las relaciones entre motivación y aprendizaje son directamente proporcionales en el éxito del logro de autonomía en los procesos de formación en los que están insertos los estudiantes. El cumplimiento de metas y la forma de evaluarlas son igualmente importantes, porque marcan rutas que generan independencia en los estudiantes. Planear momentos o etapas, no necesariamente lineales, hace parte de las estrategias que contribuyen en la mejora de aprendizajes más espontáneos, gratificantes y menos normativos (Morín, 2012; lafrancesco, 2011).

De acuerdo con las consideraciones anteriores, uno de los hallazgos encontrados en los estudiantes que participaron en el desarrollo de esta investigación es el hecho de que la mayoría afirmó no sentirse mal al momento de estudiar contenidos, lo que al relacionarlo con el bajo porcentaje de quienes en la mayoría de las ocasiones no se sienten bien $(6,6 \%)$, denota que existe un nivel importante de goce o disfrute en el ejercicio de aprendizaje. Este dato muestra que, al menos en términos del aprendizaje de conceptos o contenidos, el grueso de la población encuentra felicidad en lo que está estudiando. A propósito de este hecho los sicólogos y pedagogos que vienen estudiando las relaciones entre aprendizaje y felicidad (Csikszentmihalyi, 2006; Bisquerra, 2000; Pérez, 2014; De Zubiría, 2006) han sostenido que cuando se disfruta lo que se hace los niveles de coherencia entre el decir y el hacer se estrechan y dan pie a dos acontecimientos: por un lado se manifiestan procesos de autorregulación en la medida en que los individuos no sienten que lo que están aprendiendo se convierte en una carga, por cuanto lo disfruta como parte de su realización personal. Y por el otro, ocurre una fuerte manifestación de ética por el efecto mismo de la gratificación por cuanto, como lo sugieren las escuelas de la sicología positiva y la tradición de la Escuela de la Inteligencia Emocional, todos los aprendizajes tienden a ser autotélicos; es decir, tienen valor en sí mismos para los sujetos, y no buscan evitar un castigo o ganar un premio.

Ahora bien, el hecho de que en términos generales el $88,33 \%$ de los estudiantes encuestados se proponen generar nuevo conocimiento más que realizar ejercicios memorísticos, ratifica el hallazgo anterior, en la medida en que precisamente las relaciones entre autorregulación y aprendizaje tiene que ver con procesos críticos de construcción de conocimiento más que con repetición de contenidos, como bien lo advirtieron las distintas vertientes de la pedagogía crítica (McLaren, 1998; Freire, 2001; Apple, 2005; Giroux, 2006). Y, de hecho, los estudiantes disfrutan más cuando tienen la posibilidad de crear ideas o plantear problemas, $y$, al mismo tiempo, sufren más cuando tienen que responder cuestionarios memorísticos que los encasillan y los suelen llevar a comportamientos antiéticos, como hacer trampa en un examen o plagiar un trabajo.

De otro lado, resulta significativo que sean las mujeres de la facultad de Educación quienes manifiesten, en un porcentaje hasta del $80 \%$, que de forma autónoma recurren a métodos de aprendizaje diferentes, como una forma de autorregulación, superando de lejos los porcentajes para Contaduría (45,5\%) y Economía (40\%). Llama la atención, además, que en este último grupo de estudiantes no se presenta preferencia por la respuesta 1 (siempre). En el caso de los hombres se repite este panorama, aunque las distancias entre carreras se reducen. De esta manera, mientras que el porcentaje de estudiantes de Educación que seleccionaron las respuestas 1 y 2 llega a 73,3\%, en el caso de Contaduría y Economía alcanza 55,5\% y $60 \%$ respectivamente. Si se consideran las características específicas que presenta la facultad de Educación, resulta apenas coherente con la distancia respecto a las otras facultades. En general, los programas que tienen que ver con licenciaturas en cualquier área de la formación tienden a hacer uso de variados modelos de enseñanza y aprendizaje y continuamente están reflexionando sobre la práctica docente. Esta característica, evidentemente, motiva a los estudiantes a generar también sus propias búsquedas, especialmente porque son campos del conocimiento que pertenecen a las Ciencias Sociales y ello permite un alto grado de flexibilidad, de discusión y de planteamiento de problemas que implican creatividad y construcción del conocimiento, que muchas veces rebasan las ciencias empírico-analíticas, sin desconocerlas, como lo sugieren autores como Habermas (2008), Vásquez (2007), Bourdieu (2010), Bolívar (2007) y Becerra, Penagos \& Carrillo (2003), entre otros.

Un resultado que afloró, y que es bastante significativo 
respecto de la dimensión ética, se centra en la pregunta "creo que existe relación directa entre los valores que he aprendido en la cultura y las dinámicas de enseñanza y aprendizaje", cuya opción "la mayoría de las veces no", reflejó porcentajes que no superan el $7 \%$. Esto indica, de entrada, que los estudiantes que respondieron la encuesta hacen una valoración de la relación entre formación en la universidad y ética que tiende a hacia lo positivo. Este guarismo (cuantitativo) es un buen indicador de cómo los estudiantes efectivamente creen que los valores que son aprehendidos en los procesos culturales tienen incidencia y son relevantes en las maneras y modos como se dan los procesos de enseñanza y aprendizaje, por lo que se puede inferir que el componente ético es muy relevante en la vivencia de la formación y puede ser considerado como factor trascendente en las acciones de aprendizaje autorregulado. En ese mismo sentido, llama la atención que los hallazgos por semestre muestren que a medida que se avanza en el momento de formación, existe una mayor comprensión de que el conocimiento adquirido en la universidad tiene un componente ético capaz de incidir en la configuración como ser humano.

Esto permite inferir que la universidad, entre sus propósitos misionales, ha venido marcando pautas que los estudiantes, al menos en este estudio, reconocen como sello de su formación integral. Y es que autores como Quintero (2004) han planteado que proponer pautas para educar en valores éticos significa crear los escenarios pertinentes $y$ adecuados para que los sujetos sean capaces de internalizar los valores. Y no es porque los valores tengan necesariamente que enseñarse de manera formal, sino que se trata de cultivar todo aquello que hace posible que los valores que la sociedad ha definido como acordes para el beneficio de todos (honestidad, convivencia, solidaridad, respeto) sean vividos como parte fundamental de los procesos de formación. En sintonía con este planteamiento, Maturana y Verden-Zöller (2003) proponen que son los escenarios cotidianos emocionales y racionales los que permiten vivenciar ciertos valores que las sociedades creen que son buenos para reconocer al otro como un legítimo otro en la convivencia, por eso, fundamentalmente, se viven, y en esa medida se contagian para generar ambientes gratificantes entre los seres humanos que comparten espacios sociales y académicos. Un hallazgo de esta investigación permite corroborar este aserto: por lo menos el $81,67 \%$ de los encuestados tienen facilidad para relacionar los contenidos académicos con el comportamiento ético. De ahí se puede inferir que, por el tipo de las repuesta que se obtuvo, los impactos éticos de la educación universitaria en el comportamiento de cada individuo muestran que esta incidencia es importante para los estudiantes.
De manera general, en las tres facultades analizadas se encontró que en las relaciones entre autorregulación y aprendizaje se destaca el hecho de que las respuestas se concentran en torno a la media, reforzando la consideración de una estructura de respuestas vinculadas fuertemente a valoraciones positivas. Se ratifica, por tanto, que en cuanto al deber ser los encuestados consideran la autorregulación como un elemento importante en el aprendizaje. Al respecto, llamaron la atención las respuestas obtenidas de la pregunta "pienso que la disciplina y la organización a la hora de estudiar son un valor muy importante". En este acápite se halló que no existe diferencia sustancial entre hombres y mujeres, con el matiz de que el porcentaje de mujeres $(69,2 \%)$ que siempre consideran importantes la organización y la disciplina es mayor, tanto respecto al de los hombres $(64,7 \%)$, como al observado en el total de encuestados $(66,7 \%)$. Para el caso de la "necesidad de profundizar de manera autónoma los temas", se encuentra que la opción de respuesta 1 (siempre) tiene un comportamiento ascendente a medida que se eleva el semestre al que pertenece el estudiante. De esta manera, mientras que para los estudiantes de tercer semestre esta proporción correspondió al $25 \%$, en octavo semestre alcanza el $50 \%$, con un incremento progresivo en quinto, sexto y séptimo semestre que evidencian el 35,7\%,36,4\% y $42,9 \%$, respectivamente.

Finalmente, aunque hubo muchos hallazgos, como se pudo constatar durante el análisis de la información, cabe resalta otro más. Con respecto a si los estudiantes "se sienten afectados cuando el profesor no llega a clase y se pierde tiempo" se encontró que los porcentajes de no afectación fueron, en general, significativamente bajos. Apenas el $18,33 \%$ de toda la población contestó "siempre", mientras que el 33,33\% dice que "la mayoría de las veces sí" se siente afectada.

Esto permite inferir que la ausencia formal de profesor en algunos momentos no es una causa para desmotivar a la mayoría de los estudiantes, por cuanto crean otros espacios relacionales de socialización que se constituyen en una especie de extensión del currículo dentro de su formación integral. Ya desde la última década del siglo pasado y la primera del XXI, reconocidos pedagogos como Sacristán (2013) y Magendzo (2010), recogiendo teorías alternativas que se venían fraguando desde los años 60, comenzaron a plantear la idea de que el centro de la educación no es solamente la transmisión de contenidos preconcebidos que los estudiantes deben recibir, a manera de consignación, sino que conciben los aprendizajes también como aquellas experiencias que los sujetos comparten tanto dentro como fuera de las instituciones, los cuales tienen un fuerte componente 
socio-cultural, político, económico y académico en donde ocurren contradicciones, conflictos y toda suerte de avatares que contribuyen a la formación intelectual y moral de los individuos, como quiera que son espacios de aprendizaje permanente. $\mathrm{Y}$ a ello, por supuesto, es necesario añadirle el fuerte impacto que tiene hoy el uso de las redes sociales porque, precisamente, esto se hizo visible cuando se aplicaron las encuestas, según las cuales todos los estudiantes, sin ninguna excepción, mantuvieron contacto con otras personas a través del celular. Ello, sin duda, pudo haber influido también en el tipo de respuesta que señalaron durante el ejercicio de aplicación de la prueba. Todas estas variables contribuyen a que los estudiantes, dentro de su autonomía, puedan tomar una serie de decisiones para autorregularse.

Gran parte de los hallazgos anteriores están en relación con lo que García (2012) propone; según esta autora, los procesos de autorregulación contienen elementos motivacionales y cognitivos con una serie de componentes sistémicos que permiten afirmar que no son acciones meramente racionales que los estudiantes realizan, tal como lo promulgaban los modelos tradicionales de enseñanza. De hecho, coincide con otros autores como Bisquerra (2013); Pérez (2014); Altable (2010) y Punset (2012), quienes advierten que todos los procesos de enseñanza y aprendizaje deben ser estudiados desde una perspectiva multidisciplinar para captar los distintos factores que influyen en la autorregulación de los estudiantes: la inteligencia emocional, en donde estaría precisamente el estudio de las emociones, los estados de ánimo y, por supuesto, la motivación; cómo se comporta el cerebro frente a los escenarios formativos que incluyen contagios emocionales; los niveles de conciencia que se pueden alcanzar a través del cultivo de ciertos valores que actúan como gatilladores de autorregulación; los procesos cognitivos y metacognitivos en los que se sumerge el estudiante; los grados de madurez física e intelectual que los sujetos van logrando a medida que avanzan en su formación, y los metarrelatos (los trasfondos históricos: mitos, valores, tradiciones y normas) que traen los sujetos en formación superior de los contextos sociales, culturales y familiares de donde provienen.

\section{Conclusiones y debates}

Es importante señalar que existe un acumulado de experiencias de formación que ha contribuido a fortalecer los procesos de autorregulación en la población estudiada, y ello gracias a distintas metodologías que se han venido implementado en el interior de las facultades. Entre otras, se puede destacar el Proyecto Integrador, una experiencia pedagógica que pretende articular las distintas asignaturas en torno a una pregunta de investigación relacionada con problemas propios del contexto cultural, que se enmarca en un proyecto que, al final, los estudiantes deben sustentar delante de profesores y otros estudiantes. Esta metodología ha generado espacios de autonomía y creatividad que han provocado variadas formas de autorregulación en los estudiantes, con un fuerte componente de aprendizaje cooperativo.

En este sentido, de acuerdo con los hallazgos, se puede decir que, en general, existe una relación entre cierto comportamiento de carácter ético y los procesos de aprendizaje autorregulado. No obstante, este fenómeno se hace visible de maneras complejas y relativas a variables como la edad, el género, el semestre que se está cursando, y el tipo de programa profesional -determinado también por la jornada de estudio, que para el caso del fenómeno estudiado es nocturno-, entre otros factores.

La categoría tiempo adquiere un significado distinto para los estudiantes; del tiempo cronológico ${ }^{4}$ surge un relativo desplazamiento al tiempo kairótico ${ }^{5}$. El primero está en relación con procesos de enseñanza y aprendizaje de aquellos estudiantes que tienen un horario "regular" que les permite dedicar más tiempo a su formación, en términos de cantidad, que no está necesariamente articulada con la calidad.

Esta población, generalmente, no trabaja por distintas razones, entre otras, sus posibilidades económicas son mejores. Este no es el caso de la población objeto de esta investigación, de ahí que sea el segundo significado del tiempo (kairótico) el que tiene importancia los procesos de aprendizaje autorregulado de la población estudiada.

Existe una relación entre conciencia, trabajo, estudio y realización personal que muestra unos niveles de autorregulación relativamente altos en las tres facultades estudiadas, lo cual está en relación con la tendencia a percibir el estudio más como una experiencia gratificante que como una actividad obligatoria, y eso marca pautas de autorregulación significativas tanto en hombres como en mujeres, a pesar de que estas últimas presentan un porcentaje levemente más alto.

Lo anterior muestra un rasgo que vincula la autorregulación directamente con la motivación, lo que confirma la tesis consuetudinaria que han afirmado

\footnotetext{
4. Del griego хроvо入оүıкóc, cuyo significado está en relación con el tiempo convencional, medido, controlado; dentro del cual surgen diversos acontecimientos que marcan pautas de comportamiento. 5. Del griego кaıрóc, que se relaciona con una experiencia existencial, que tiene que ver más con la experiencia y su significado en cada momento, que con la linealidad del tiempo formal.
} 
de la mayoría de las escuelas modernas de pedagogía, que sostienen que la motivación es un eje decisivo en los procesos de enseñanza y aprendizaje. Aunado a ello, hay valores éticos involucrados en la experiencia cuya expresión tiene fuentes diversas, como la familia, el ambiente cultural, las convicciones personales y el legado ético de la institución como tal, que marcan pautas de comportamiento autorregulado. Este último aspecto relaciona de facto "la universidad y el aula como un recinto sagrado donde se gesta el nacimiento de personas libres mediante la interacción de maestros y estudiantes"(García \& González, 2015). No obstante, como elemento de discusión que abre las puertas para seguir investigando el fenómeno, queda pendiente determinar cuál es el alcance de los niveles de autorregulación encontrados frente a la calidad del rendimiento académico. Esto en razón a que el hecho de que los estudiantes manifiesten niveles de autorregulación e incluyan valores en su proceso de formación no conduce necesariamente a generar excelencia académica.

En términos de aportes a la educación, a nivel nacional e internacional, se puede decir que los resultados de este trabajo de investigación muestran posibles rutas para seguir fortaleciendo la capacidad de autorregulación de los estudiantes, a saber: fortalecer los procesos de formación de la conciencia crítica como factor fundamental que alimenta las capacidades de decisión de los sujetos frente a su proceso de formación. A ello es necesario articular la inteligencia emocional para desarrollar el sentimiento, la voluntad y los estados de ánimo positivos que permitan generar ambientes apropiados para el ejercicio de la autorregulación. También se sugiere crear mecanismos para desarrollar lo que Gardner (2005) Ilama mente disciplinada, como una de las mentes que se requieren, según él, para una educación en el siglo XXI en dos sentidos, el primero, señalado por el autor, en la perspectiva de articular los distintos campos del conocimiento para dialogar con ellos e integrarlos en los procesos de apropiación del conocimiento. El segundo, resignificado por el investigador (Burgos, 2016), es crear escenarios de corresponsabilidad académica dentro de ejercicios permanentes de autodisciplina y control de todos los procesos para garantizar, de esa manera, que las misiones de las instituciones de educación superior se vayan haciendo visibles, con la participación activa de los estudiantes que cultivan capacidades de autodirección, en donde las normas son un medio y no un fin.

\section{Referencias}

Albornoz, M. (2009). Educación basada en competencias y constructivismoun enfoque y un modelo para la formación pedagógica del siglo XXI (No. F/378 B4).

Altable, C. (2010). Los senderos de Ariadna. Transformar las relaciones mediante la coeducación emocional. Barcelona, Granada: Octaedro.

Apple, M. W. (2005). Entre el neoliberalismo y el neoconservadurismo: educación y conservadurismo en un contexto global. In Globalización y educación: manual crítico (pp. 59-78). Editorial Popular.

Becerra, A. J., Penagos, R. Á., \& Carrillo, A. T. (2004). La práctica investigativa en ciencias sociales. U. Pedagógica Nacional.

Bisquerra, R. (2000). Educación emocional y bienestar.

Bisquerra, R. (2013). Cuestiones sobre bienestar. Madrid: Wolters Kluwer.

Bolívar, A. (2007). Educación para la ciudadanía: algo más que una asignatura. Graó.

Bourdieu, P. (2010). La sociología es un arte marcial. Madrid: Editora Gisele Sapiro.

Burgos, F. A. (2016). Los escenarios educativos informales como espacios de inclusión y calidad de vida de menores en situación de marginación y desplazamiento. (Tesis doctoral inédita). Universidad de Sevilla, Sevilla.

Campo, R.\& Restrepo, M. (2008) Formación integral. Bogotá: PUJ. Camarero, F. \& Suárez, M. (2014). Estilos y estrategias de aprendizaje en estudiantes universitarios. Psicothema, 12(4), 615-622: Universidad de Oviedo.

Cascante, J. (2011). Métodos mixtos de investigación. Guía de estudio. Universidad Estatal a Distancia, Institución Benemérita de la Educación y Cultura.

Csikszentmihalyi,M.(2006). Theexperienceofpsychopathology: Investigating mental disorders in their natural settings. Cambridge University Press.

De Zubiría, J. (2006). Los modelos pedagógicos: hacia una pedagogía dialogante. Coop. Editorial Magisterio.

Di Gresia, L. (2007). Rendimiento académico universitario. Versión modificada de un capítulo de tesis doctoral. Universidad Nacional de La Plata. digresia. com. ar/files/ rendacadaaep/rendacadaaep_full.pdf

Erazo, O. (2012). El rendimiento académico, un fenómeno de múltiples relaciones y complejidades. Revista Vanguardia Psicológica Clínica Teórica y Práctica, 2(2).

Freire, P. (2001). Pedagogía dela indignación. Ediciones Morata.

García, M. (2012). La autorregulación académica como variable explicativa de los procesos de aprendizaje universitario. Revista de Currículum y Formación de Profesorado, 16(1), 203-221.

García, S. E. \& González, J. (2015). La universidad educa para la libertad. Reflexiones de un humanismo personalista. I+DRevista de Investigaciones, 5(1), 108-123. Recuperado de http://www.udi.edu.co/revistainvestigaciones/index. php/ID/article/view/11

Garbanzo, G. (2007). Factores asociados al rendimiento académico. Revista de la Universidad de Costa Rica, 9(2).

Gardner, H. (2005). Las cinco mentes del futuro: un ensayo educativo (No. L1546). Paidos,. 
Garzón, R., Rojas, M. O., Riesgo, L. D., Pinzón, M., \& Salamanca, A.L. (2010). Factores que pueden influir en el rendimiento académico de estudiantes de Bioquímica que ingresan en el programa de Medicina de la Universidad del Rosario-Colombia. Educación médica, 13(2), 85-96.

Giroux, H. (2006). America on the edge: Henry Giroux on politics, culture, and education. Springer.

Habermas, J. (2008). Teoría de la Acción Comunicativa. Madrid: Editorial Trotta.

lafrancesco, G. M. (2011). Algunas problemáticas de la investigación en educación y pedagogía en Colombia: estrategias para enfrentarlas y resolverlas. Revista de Investigación, Desarrollo e Innovación, 1(2), 7-16.

Ibáñez, I. (2014). El delirio del capitalismo. Madrid: Siglo XXI.

Magendzo, A. (2010). Curriculum, Educación para la democracia en la modernidad. Bogotá: PIEE Editores.

Maldonado, C. (2005). Ética decisión racional y teoría de la acción. En Problemas de ética aplicada: Universidad el Bosque.

Martín, I. (2007). Estrés académico en estudiantes universitarios. Apuntes de Psicología, 25(1), 87-99.

Maturana, H. R., \& Verden-Zöller, G. (2003). Amor y juego: fundamentos olvidados de lo humano, desde el patriarcado a la democracia. JC Sáez Editor.

Morin, E. (1999). Los siete saberes necesarios para la educación del futuro. Organización de las Naciones Unidas para la Educación, la Ciencia y la Cultura. París.

Morin, E. (2012). La vía para el futuro de la humanidad. Reis, 140, 173-184.

McKeachie, W. J., Pintrich, P. R., Lin, Y., \& Smith, D. (1986). Teaching and learning in the college classroom. Ann Arbor, MI: University of Michigan.

McLaren, P. (1998). Multiculturalismo revolucionario: pedagogías de disensión para el nuevo milenio. Siglo XXI.

Nussbaum, M. (2010). Sin fines de lucro. Bogotá: Kats, discusiones.

Páramo, B. P. (2010). La investigación en las Ciencias Sociales. Técnicas derecolección deinformación. Bogotá:Universidad Piloto de Colombia.

Pereira, Z. (2010). La mirada de estudiantes de la Universidad Nacional hacia el docente y la docente: sus características y clima de aula. (Tesis doctoral sin publicar). Universidad Estatal a Distancia. San José, Costa Rica.

Pérez, T. (2014). El poder transformador de los educadores. Bogotá: Magisterio.

Pintrich, P. R., Smith, D. A., García, T., \& McKeachie, W. J. (1991). A manual for the use of the motivational strategies for learning questionnaire (MSLQ). Ann Arbor, MI:University of Michigan, National Center for Research to Improve Postsecondary Teaching and Learning.

Punset. E. (2012). Lo que nos pasa por dentro. Madrid: Destino Editorial.

Quintero. M. (2004). ¿Qué significa educar en valores hoy? Bogotá: Octaedro OEl.

Sacristán, J. (2013). En busca del sentido de la educación. Madrid: Ediciones Morata.
Salcedo, A. (2010). Deserción universitaria en Colombia. Rev Academia Virtualidad, 3(1), 50-60.

Sampieri, H., Collado, C. F. \& Lucio, P. B. (2006). Metodología de la investigación. MacGraw-Hill. Chile Smith, $M$

Serrano, S. (2015). La relación entre el habitar-ethos y la ética. Antropología educativa. I+ D Revista de Investigaciones, 6(2), 6-18.

Tejedor, F. \& García, A. (enero-abril, 2006). Causas del bajo rendimiento del estudiante universitario (en opinión de los profesores y alumnos). Propuestas de mejora en el marco del EEES. Revista de Educación, 342, 443-473.

Vásquez, F. (2007). Educar con maestría. Bogotá: Universidad de la Salle

Vasco, C. (2003). Posmodernidad, Ciencias y Educación. Bogotá: Javegraf.

Vives, M. (2015). Confianza. Propuesta de un modelo teórico sobre su génesis y consolidación. Bogotá: Universidad de la Salle.

Zimmerman, B. (1989). Evaluación de la autoeficacia regulatoria: una perspectiva social cognitiva. Evaluar, (5). 